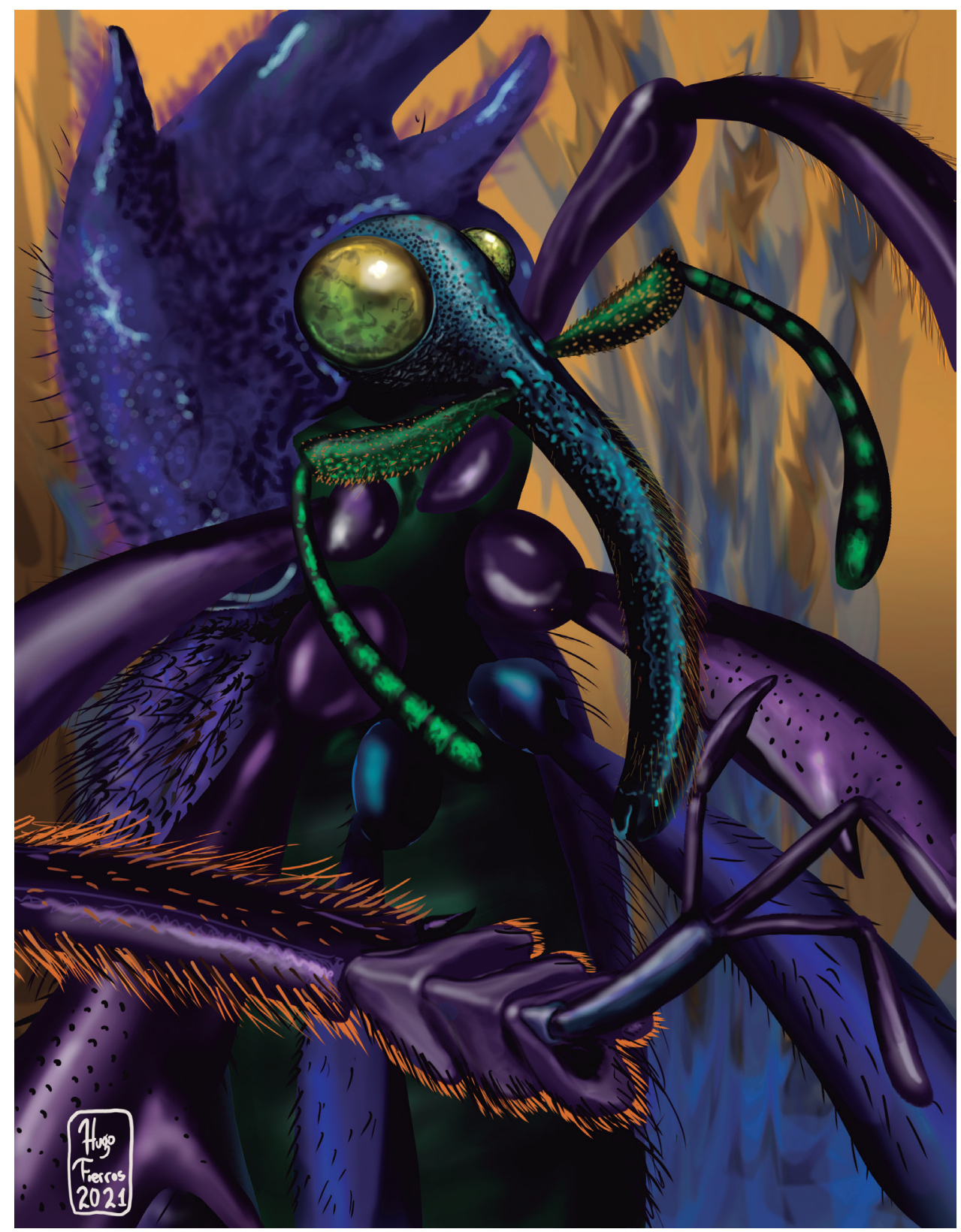

Dugesiana, Año 28, No. 2, (julio-diciembre, segundo semestre de 2021), es una publicación semestral, editada por la Universidad de Guadalajara, a través del Centro de Estudios en Zoología, por el Centro Universitario de Ciencias Biológicas y Agropecuarias. Camino Ramón Padilla Sánchez \# 2100, Nextipac, Zapopan, Jalisco, Tel. 37771150 ext. 33218, http://148.202.248.171/dugesiana/index.php/DUG/index, glenusmx@gmail.com. Editor responsable: José Luis Navarrete-Heredia. Reserva de Derechos al Uso Exclusivo 04-2009-062310115100-203, ISSN: 2007-9133, otorgados por el Instituto Nacional del Derecho de Autor. Responsable de la última actualización de este número: José Luis Navarrete-Heredia, Editor y Ana Laura González-Hernández, Asistente Editorial. Fecha de la última modificación 1 de julio de 2021, con un tiraje de un ejemplar.

Las opiniones expresadas por los autores no necesariamente reflejan la postura del editor de la publicación.

Queda estrictamente prohibida la reproducción total o parcial de los contenidos e imágenes de la publicación sin previa autorización de la Universidad de Guadalajara. 


\title{
Estructura areográfica y efectos del cambio de uso de suelo en especies endémicas de la Faja Volcánica Transmexicana
}

\author{
Areographical structure and effects of the land use change in the endemic species of the Transmexican \\ Volcanic Belt \\ Karla Y. Aguilar-Ortega ${ }^{1} \&$ Tania Escalante ${ }^{1 *}$ \\ ${ }^{1}$ Grupo de Biogeografía de la Conservación, Departamento de Biología Evolutiva, Facultad de Cien- \\ cias, Universidad Nacional Autónoma de México (UNAM), Circuito Exterior s/n, Ciudad Universita- \\ ria, 04510 Ciudad de México, México; ${ }^{1}$ tescalante@ciencias.unam.mx, ORCID: https://orcid.org/0000- \\ 0002-9127-8969; 'karla.yao05@gmail.com,ORCID: https://orcid.org/0000-0002-0969-1165; *Autor de \\ correspondencia: tescalante@ciencias.unam.mx
}

\begin{abstract}
RESUMEN
Los estudios sobre las áreas de distribución geográfica (ADG) proporcionan información sobre cómo se establecen las especies en el espacio y tiempo, y los efectos del cambio de uso de suelo sobre los patrones geográficos de la biodiversidad. En la provincia biogeográfica de la Faja Volcánica Transmexicana (FVT) habita un alto número de especies endémicas, a la vez que presenta altas tasas de deforestación. En el presente trabajo: (a) se describen cuantitativa y cualitativamente las ADG histórica de 167 especies de anfibios, aves, insectos, mamíferos y plantas endémicas de la FVT, y (b) se analiza el efecto del cambio de uso de suelo actual en esas distribuciones geográficas. En general, la clase Mammalia tuvo las mayores superficies ocupadas, y fue principalmente de distribución continental (las especies se distribuyen en más de una región biogeográfica). Por otro lado, la mayoría de las especies de todos los grupos taxonómicos fueron transicionales y discontinuas. Considerando el cambio en el uso del suelo, el $85 \%$ de las especies tuvo cambios en sus ADG históricas, de las que dos tercios tuvieron una pérdida mayor al 50\%. Los grupos taxonómicos más afectados por pérdida total de su ADG histórica, fueron Insecta y Amphibia. La mayoría de las especies ocuparon altitudes elevadas, y la superficie perdida se localizó mayoritariamente entre los 1,500 y 3,000 msnm. La variabilidad en el comportamiento distribucional de las especies sugiere patrones internos y diferenciados en la FVT, y mayor complejidad biológica, geológica y evolutiva de la esperada, con múltiples relaciones de la FVT con las provincias adyacentes. Los estudios areográficos pueden constituir un punto de partida para la preservación de áreas no transformadas, que cumpla el objetivo de prevenir la posible extirpación de las especies.

Palabras clave: anfibios, área de distribución geográfica, aves, insectos, mamíferos, plantas, Zona de Transición Mexicana.
\end{abstract}

\begin{abstract}
The studies about the geographic distribution areas (GDA) provide information on how species are established in space and time, and the effects of land use change on geographic patterns of biodiversity. In the Trans-Mexican Volcanic Belt (TVB) biogeographic province inhabits a high number of endemic species; as well as it has high deforestation rates. In this manuscript: (a) the historical GDA of 167 endemic species of amphibians, birds, insects, mammals and plants of the TVB are quantitatively and qualitatively described, and (b) we analyze the effect of the change in the current land use in those geographic distributions. In general, the class Mammalia had the largest occupied surfaces; the highest average of the three magnitudes and mainly of continental distribution (the species are distributed in more than one biogeographic region). On the other hand, most of the species of all taxonomic groups were transitional and discontinuous. Considering the land use change, $85 \%$ of the species had changes in their historical GDA; two thirds of them with a loss greater than 50\%. The taxonomic groups most affected due to total loss of its historical ADG were Insecta and Amphibia. Most of the species occupied higher altitudes and, therefore, these altitudinal ranges had the greatest loss of surface area between 1,500 and 3,000 masl. The variability in the distributional behavior of the species suggests internal and differentiated patterns into the FVT, and greater biological, geological and evolutionary complexity than expected, with multiple relationships of the FVT with the adjacent provinces. The areographic studies could constitute a starting point for the preservation of non-transformed areas that fulfills the objective of preventing the possible extirpation of the species.
\end{abstract}

Keywords: amphibians, geographic distribution areas, birds, insects, mammals, plants, Mexican Transition Zone.

El área de distribución geográfica (ADG) de una especie es aquella porción del espacio geográfico en la que tal especie está presente y con la que interactúa de manera no efímera (Zunino y Palestrini 1991). El estudio de la forma, tamaño y distribución espacial de las ADG constituye a los análisis areográfícos o corológicos (Rapoport y Monjeau
2001), a partir de los cuales se obtiene información acerca de la biodiversidad, lo cual contribuye a la delimitación de regiones biogeográficas (Kohlman y Sánchez 1984).

Una forma de inferir las ADG de las especies es mediante modelos de distribución empíricos. Estos modelos permiten relacionar las observaciones de campo con va- 
riables de predicción ambiental (Guisan y Zimmermann 2000), produciendo así mapas de distribución potencial. El área de distribución potencial puede indicar la presencia de la especie analizada en sitios donde la especie no está presente, dado que existen otros factores, como por ejemplo, los patrones del uso del suelo, que no están considerados en la modelación (Sánchez-Cordero et al. 2004).

No existe una metodología estándar para cuantificar los tamaños de las ADG, sin embargo, es común usar la magnitud lineal máxima (distancia en línea recta entre los dos sitios ocupados más separados), la magnitud latitudinal (distancia en línea recta entre los sitios ocupados más separados de forma latitudinal) y la magnitud longitudinal (distancia en línea recta entre los sitios ocupados más separados longitudinalmente) (Gaston 1996). Por otro lado, las ADG pueden clasificarse con base en su extensión, situación geográfica y su continuidad (Cabrera y Willink 1973; Gaston 1996). La extensión se refiere a la superficie ocupada en términos relativos o absolutos, pudiendo ser regional o continental (si abarca una región biogeográfica o un continente, respectivamente; Cabrera y Willink 1973). De acuerdo con su situación geográfica, las ADG también pueden clasificarse como polares, holáricas, tropicales, pantropicales, paleotropicales, neotropicales y australes (Cabrera y Willink 1973). Finalmente, las áreas pueden ser continuas si son homogéneas e ininterrumpidas (si constituyen una sola mancha o un solo polígono a cierta escala) o discontinuas (Cabrera y Willink 1973).

La condición de continuidad, discontinuidad y disyunción natural de las ADG ha sido alterada de manera antrópica principalmente por el cambio de uso de suelo (CUS). El CUS es un proceso que implica la conversión de los paisajes naturales, el cual ha transformado gran proporción de la superficie terrestre (Foley et al. 2005) y es uno de los principales efectos negativos de las actividades humanas (Morrone y Escalante 2016). Las áreas transformadas incluyen principalmente a la agricultura, la vegetación inducida, las plantaciones forestales, la deforestación y los asentamientos humanos (Velázquez et al. 2003; Figueroa y Sánchez-Cordero 2008). El CUS ha dado como resultado una reducción y fragmentación significativas de los hábitats naturales, lo que a su vez ha reducido el ADG de especies a subconjuntos de sus áreas potenciales (Sánchez-Cordero et al. 2004). Yáñez-Arenas et al. (2012) consideran que la mayoría de los subconjuntos de áreas potenciales (parches) no poseen el área suficiente para soportar poblaciones mínimas viables a largo plazo. En particular, las especies endémicas son más propensas a la extirpación al contar sólo con pequeños fragmentos de hábitat no transformado (Sánchez-Cordero et al. 2005).

Un caso de especial interés por el alto número de especies endémicas en México es la provincia biogeográfica de la Faja Volcánica Transmexicana (FVT) (Morrone et al. 2017; Fig. 1). La FVT es una cadena de volcanes con orientación este-oeste que se extiende desde la vertiente del Pacífico hasta la del Golfo de México (Ferrusquía-Villafranca
2007) y se encuentra localizada entre los $18^{\circ} 30^{\prime}$ y $21^{\circ} 30^{\prime}$ de latitud norte, por arriba de los 1,800 metros sobre el nivel del mar (Morrone 2017). La FVT ha experimentado un aumento en deforestación y urbanización desde la década de 1960, convirtiendo el 70\% del hábitat no transformado en agrosistemas y asentamientos rurales o urbanos (Toledo et al. 1989; Challenger 1998; Arriaga et al. 2000), ya que es una región del país en donde se concentran varios de los núcleos urbanos más importantes de México (Cantellano 2007; González-Ávila et al. 2018). En el año 2014 se registró el $62.2 \%$ de cambio en la vegetación original debido a las actividades antrópicas, y desde entonces la tasa de deforestación anual ha aumentado significativamente (Suárez-Mota y Téllez-Valdés 2014). Sorprendentemente, son escasos los estudios de los cambios en los patrones de distribución de las especies debidas al CUS.

Puesto que a la fecha se desconoce el efecto del CUS en las ADG de las especies endémicas de la Faja Volcánica Transmexicana, el presente estudio tiene como objetivos: (a) describir cuantitativa y cualitativamente las áreas de distribución geográfica histórica de 167 especies de anfibios, aves, insectos, mamíferos y plantas endémicas, y (b) analizar el efecto del cambio de uso de suelo actual en las distribuciones geográficas de estas especies. Al respecto, esperamos que: independientemente de los grupos taxonómicos, las especies presentarán patrones de distribución compartidos, y los sitios de hábitat no alterado de las ADG se observarán a altitudes mayores, debido que la actividad antrópica generalmente se encuentra representada en altitudes bajas.

\section{MATERIALES Y MÉTODOS}

Datos. Las ADG de 167 especies endémicas de la FVT fueron obtenidas a partir de Escalante et al. (2020; Cuadro S1 del Material suplementario). Las 167 especies fueron seleccionadas con ayuda de los especialistas en los grupos taxonómicos puesto que tuvieron al menos el 50\% de sus registros distribucionales ubicados en la FVT (para más detalles, ver Escalante et al. [2020]). Estas especies incluyen a 25 anfibios (Flores-Villela et al. 2010), un ave (Navarro-Sigüenza et al. 2003; Peterson et al. 2016), 89 insectos (Reyes-Castillo y Morón Ríos 2005; SNIB-Conabio 2017; GBIF 2017), 15 mamíferos (Escalante 2015a) y 37 plantas (Escalante 2015a; Hernández y Gómez-Hinostrosa 2011, 2015; Téllez-Valdés 2017). Las 167 ADG consideradas como áreas históricas de las especies fueron inferidas mediante modelos de distribución potencial o mediante un buffer de $2 \mathrm{~km}$ alrededor de las localidades de registro. Los modelos de 42 especies se obtuvieron con el algortimo de máxima entropía en el paquete 'dismo' (Hijmans et al. 2017; R Core Team 2017), con salida logística. Los detalles pueden ser consultados en el material suplementario de Escalante et al. (2020). Por otro lado, 125 especies no pudieron ser modeladas, puesto que no tuvieron registros suficientes o cuyos modelos no pasaron la validación. Estas especies representan a la mayoría de las especies y tienen 
gran importancia biogeográfica y de conservación por su rareza geográfica. Para incluirlas en el análisis, las ADG de estas especies fueron inferidas con un buffer de $2 \mathrm{~km}$ alrededor de cada localidad, como sugiere la Unión Internacional para la Conservación de la Naturaleza para el cálculo del área de ocupación (IUCN 2019).

Areografía. La superficie histórica ocupada para cada especie se calculó en hectáreas utilizando una proyección Cónica Equivalente de Albers con valores para México. Con fines comparativos, se calculó la media y desviación estándar para cada clase taxonómica.

La magnitud máxima lineal se calculó a partir de la distancia máxima entre los vértices de los polígonos convexos de cada una de las ADG. Para determinar las magnitudes longitudinales y latitudinales se consideraron las coordenadas máximas y mínimas, delimitando el área de distribución dentro de un cuadro, donde las distancias verticales corresponden a la magnitud latitudinal y las distancias horizontales a la magnitud longitudinal.

Posteriormente, las 167 ADG se clasificaron de acuerdo con su extensión, situación geográfica, continuidad y ocupación con base en la clasificación propuesta por Cabrera y Willink (1973). No obstante, se adecuaron los tipos de áreas considerando las características que presenta México, clasificándolas de acuerdo con su extensión, continuidad y ocupación tomando como referencia las 14 provincias biogeográficas propuestas por Morrone et al. (2017). Dado que la clasificación de las ADG de acuerdo con su situación geográfica de Cabrera y Willink (1973) no es informativa para las 167 especies, y que recientemente la FVT ha sido descrita como parte de la Zona de Transición Mexicana (Morrone et al. 2017), se propuso una clasificación para México. Todos los análisis areográficos se realizaron en QGIS v.2.18 (QGIS 2018).

Cambio de uso de suelo. A partir de la Serie VI de Uso del Suelo y Vegetación de INEGI (2017a, 2017b), que presenta la distribución de los diferentes tipos de vegetación natural e inducida, así como la ubicación de las áreas agrícolas de México, se extrajeron los polígonos de CUS (agricultura, asentamientos humanos, vegetación cultivada y vegetación inducida) de la FVT (Morrone et al. 2017; Fig. 1). Estos polígonos se intersectaron con las 167 ADG históricas, para calcular la pérdida de cobertura vegetal original en hectáreas, así como la superficie de área no transformada. A estas ADG resultantes se les llamó ADG actuales.

Para evaluar el efecto del CUS en el desplazamiento altitudinal de las especies, se utilizó el mapa de Hipsometría de INEGI (1990-1992). Cada intervalo hipsométrico se intersectó con cada una de las ADG históricas y actuales, con la finalidad de obtener su superficie en hectáreas y analizar el cambio entre las ADG históricas con las actuales.

\section{RESULTADOS}

Areografía. La superficie histórica ocupada para cada especie se presenta en el Cuadro S1 del Material suplementario. En general, la clase Mammalia tuvo las mayores superficies ocupadas, sin embargo, la clase Magnoliopsida mostró mayor variación en tamaños de las ADG (Cuadro 1).

Los promedios de las magnitudes máxima lineal, latitudinal y longitudinal de las 167 ADG por clases taxonómicas se presentan en el Cuadro 2. Aunque la clase Aves solo presenta un dato, este es parecido a los intervalos de las medias de las otras clases. Para la clase Mammalia, el promedio de las magnitudes máxima lineal, latitudinal y longitudinal fueron las más grandes, teniendo en general áreas de distribución más amplias.

La clasificación de las áreas de acuerdo con su extensión, situación geográfica, continuidad y ocupación se presenta en el Cuadro S2 del Material suplementario. De acuerdo con los tipos de extensión de Cabrera y Willink (1973) y la regionalización de Morrone (2015), 86 especies $(51.5 \%)$ se consideraron regionales porque abarcan solo una región biogeográfica y las restantes fueron clasificadas como continentales (ocupando un solo continente pero más de una región). La mayoría de los mamíferos (86.67\% de las especies) tuvo una distribución continental, al contrario de los insectos (31.47\%).

La nueva clasificación de la situación geográfica para México, con base en Morrone et al. (2017), se presenta en el Cuadro 3. Con esta modificación, las especies pudieran pertenecer exclusivamente a solo una de las regiones: Neártica, Neotropical o Transicional (referida a la Zona de Transición Mexicana), aunque también habría posibilidad de especies con patrones compartidos entre dos o más regiones (Neártico-Transicional, Neártico-Transicional-Neotropical y Transicional-Neotropical). De esta manera, la mayoría de las especies se caracterizó como Transicional (86 especies, $51.5 \%)$, siete especies $(4.19 \%)$ correspondieron al tipo Neártico-Transicional, 40 especies $(23.95 \%)$ del tipo Neártico-Transicional-Neotropical y 33 especies Transicional-Neotropical $(19.76 \%)$. Ninguna especie fue exclusivamente Neártica y solo la ADG de un díptero se consideró Neotropical. La clase con más especies transicionales fue Insecta con el $67.41 \%$ de su total de especies, seguida de Amphibia con el 48\%.

Respecto a su continuidad, la mayoría de las especies mostró áreas de distribución discontinuas (108 especies, $64.67 \%$ ). Todas las clases tuvieron áreas continuas, excepto Aves, predominando los insectos con 28 especies (16.76\%).

Finalmente, la ocupación en unidades biogeográficas (Gaston, 1996; Morrone et al. 2017) resultó en 76 especies (45.50\%) de distribución restringida a la FVT, tres especies estuvieron presentes solo en la provincia de la Sierra Madre del Sur $(1.79 \%)$ y una en la provincia de la Cuenca del Balsas $(0.59 \%)$. Las especies catalogadas de distribución amplia fueron 87 (52.09\%). De las especies de distribución amplia, 40 están presentes en cuatro o más provincias y nueve en tres provincias. De las restantes, 22 especies comparten su distribución entre la FVT y la Cuenca del Balsas, cinco entre la FVT y la provincia Veracruzana, tres con el Desierto Chihuahuense, dos con la provincia de las Tierras 
Bajas del Pacífico, dos con la Sierra Madre Occidental, dos con la Sierra Madre Oriental y dos con la Sierra Madre del Sur. Respecto a los grupos taxonómicos, la mayoría de los insectos tuvo distribución restringida (64\%), alrededor de la mitad de los anfibios, un cuarto de las especies de plantas y solo dos especies de mamíferos.

Cambio de uso de suelo. De las 167 especies analizadas, el $4.79 \%$ no mostró ningún cambio, el 10.18\% presentó pérdida total del ADG con vegetación original y la mayoría (85.02\%) tuvo cambios en sus ADG históricas (Fig. 2a; Cuadro S1 del Material suplementario). Con respecto a las 142 especies cuyas ADG históricas fueron transformadas, dos tercios tuvieron una pérdida mayor al $50 \%$ de la vegetación original, mientras que un tercio perdió menos del 50\% (Fig. 2b). Solo siete especies de insectos y una planta no tuvieron superficie transformada. Los grupos taxonómicos que presentaron pérdida total de su ADG histórica, fueron Insecta con 16 especies y Amphibia con una especie. Por otro lado, la mayoría de las plantas y anfibios, y aproximadamente la mitad de las especies de insectos, tuvieron más del $50 \%$ de pérdida de vegetación original en sus ADG, así como 12 de las 15 especies de mamíferos. La única especie de ave perdió el $42.01 \%$ de su ADG histórica.

La mayoría de las especies ocuparon altitudes mayores, entre los 1,500 y 3,000 msnm (Cuadro 4). El 34.63\% de la superficie ocupada en las ADG estuvo entre los 2,000 a 2,500 msnm, es decir, en las altitudes intermedias; seguido por el intervalo de 1,500 a $2,000 \mathrm{msnm}$ con el $25.96 \%$ y de los 2,500 a 3,000 por el $19.93 \%$. Los intervalos altitudinales con mayor superficie perdida por el CUS se presentaron entre los 1,500 y 3,000 msnm (Fig. 4).

El intervalo altitudinal en el que se perdió mayor superficie para las especies de mamíferos fue el de 2,500 a 3,000 $\mathrm{m}$, donde el ADG histórica se ha reducido en este intervalo hasta en un $60 \%$. Lo mismo ocurre en los anfibios, el ave y las plantas; sin embargo, la mayor pérdida para los insectos ocurrió en el intervalo de los 2,000 a 2,500 m, donde solo se conserva el $32.78 \%$ de la superficie histórica en las ADG reales.

\section{DISCUSIÓN}

Areografía. A pesar de que la areografía no es una disciplina reciente (Rapoport 1975), existen pocos estudios areográficos comparativos en México. Aunado a lo anterior, las diferentes maneras de clasificar a las ADG pueden conducir a estudios no comparables, y las múltiples clasificaciones en ocasiones requieren ser adaptadas a los contextos de cada análisis, como en este trabajo.

En la FVT, las ADG de las especies endémicas presentaron gran variedad de tamaños entre clases taxonómicas. A pesar de que las especies analizadas tienen una gran parte de su distribución restringida a la FVT o han sido reconocidas como especies endémicas, su comportamiento distribucional en cuanto a su superficie y magnitudes fue variable. Esta variabilidad también se observó en su situación geográfica, donde se esperaba que la mayoría solo perteneciera a la Zona de Transición Mexicana, en particular a la FVT. Esto puede ser evidencia de varias hipótesis que discutimos más adelante: (1) las ADG históricas generalmente están sobreestimadas por los modelos de distribución potencial; (2) existen patrones de distribución geográfica internos y diferenciados en la FVT; (3) los límites de algunas unidades de las regionalizaciones biogeográficas actuales son difusos y posiblemente deben revisarse; y (4) hay mayor complejidad biológica, geológica y evolutiva de la esperada, con múltiples relaciones de la FVT con las provincias adyacentes.

Respecto a nuestra primera hipótesis, es posible que las ADG de las especies modeladas estén sobrepredichas, dado que estos modelos representan un espacio ambiental y geográfico en donde se postula que la especie podría ocupar. Sin embargo, estos modelos basados en el concepto de nicho ecológico, solo representan algunas condiciones ambientales óptimas (las que se emplearon en el proceso de modelado), pero omiten otras variables ambientales, ecológicas e histórico-evolutivas que moldean a las ADG (Illoldi y Escalante 2008; Soberón et al. 2017).

La segunda hipótesis sugiere que existen patrones biogeográficos internos en la FVT, resultado de las diferencias entre las ADG de las especies, las cuales podrían conformar patrones de endemismo como se ha encontrado en trabajos previos (por ejemplo: Gómez-Tuena et al. 2005; Ferrari et al. 2012; Gámez et al. 2012; Suárez-Mota et al. 2013). Morrone (2020) representó parte de estos hallazgos en una regionalización en la que se distinguen dos subprovincias y cuatro distritos. Adicionalmente, el reconocimiento de cenocrones - taxones que comparten una misma historia biogeográfica - analizados mediante un rebanado temporal (Corral-Rosas y Morrone 2017), permitirá conocer las relaciones internas de las unidades menores de la FVT.

La tercera hipótesis está muy relacionada con la segunda, puesto que la regionalización biogeográfica natural tiene como patrón subyacente al endemismo y a la monofilia de las áreas (Escalante 2017). Las unidades de las regionalizaciones biogeográficas tienen límites difusos, los cuales aún son poco entendidos y estudiados (Noguera-Urbano y Ferro 2018), por lo que establecimiento de líneas fijas en los mapas representa un reto conceptual y metodológico que debe ser revisado, en particular para provincias que forman parte de Zonas de Transición, como la FVT. Los resultados obtenidos en este trabajo también pueden ser evidencia de los diferentes conceptos de endemismo que pueden ser cuestionados (por ejemplo: cuasi-endémico, semi-endémico), y la importancia del endemismo como un concepto relativo (Escalante 2015b; Noguera-Urbano 2017; Fattorini 2017).

La complejidad biótica y geológica de la FVT ha sido estudiada desde diferentes puntos de vista (ver Morrone 2020), sin embargo nuestros hallazgos nos permiten proponer una cuarta hipótesis, donde esta complejidad es mayor que la esperada. La FVT se encuentra en una zona de transición entre dos regiones, por lo cual muchas especies extienden su distribución hacia una u otra región, o hacia ambas. 
Las provincias a las que más frecuentemente extendieron su distribución geográfica las especies aquí analizadas fueron las neotropicales, particularmente hacia la Cuenca del Balsas. Algunos autores han propuesto que esta cuenca podría pertenecer a la Zona de Transición Mexicana (por ejemplo: Arriaga et al. 1997; Morrone et al. 1999), pero recientemente y con mayor evidencia, otros autores sostienen que forma parte de la región Neotropical en el sentido estricto (Morrone 2014; Morrone et al. 2017). La evidencia proporcionada por el presente estudio, parece indicar que existe una relación estrecha entre la FVT y la Cuenca del Balsas, al menos en una porción de la FVT, ya que se ha postulado que la misma FVT no constituye una unidad homogénea, probablemente resultado de la asincronía de su origen geológico (Ferrari et al. 2012; Gámez et al. 2012). Así mismo, Espinosa et al. (2008) señalaron la existencia de diferentes taxones de plantas y animales que habitan en las laderas de la FVT y la Sierra Madre del Sur orientadas hacia la Cuenca del Balsas.

Cambio de uso de suelo. El cambio de uso de suelo ha mostrado gran impacto en la biodiversidad a nivel mundial (Sánchez-Cordero et al. 2004; Daskalova et al. 2020). Aunque en los últimos años en México es posible que se esté deteniendo el cambio de uso de suelo debido a la deforestación, siguen ocurriendo otras transiciones mayores como la transformación hacia áreas urbanas (Moreno-Sánchez et al. 2017; Gao et al. 2018; Bonilla-Moheno y Aide, 2020). En particular, Aguilar-Tomasini et al. (2020) encontraron que la mayoría de las áreas naturales protegidas de la FVT han sido poco efectivas para detener el CUS. Dentro de la misma FVT, las áreas naturales protegidas mostraron diferentes transiciones, muchas de ellas tendiendo hacia la conversión a la agricultura (Aguilar-Tomasini et al. 2020).

Los resultados obtenidos aquí indican grave pérdida de las ADG históricas por el CUS. Sánchez-Cordero et al. (2009) alertaron que la pérdida del hábitat hasta en el 90\% de las ADG de las especies puede conducir a alto riesgo de extinción. Otros efectos del CUS incluyen repercusiones en la dinámica ecosistémica, clima, regímenes de incendios e inundaciones, carga de sedimentos y fertilización con nitrógeno en ecosistemas de agua dulce, invasión de especies y patógenos, entre otros (Hansen et al. 2012). La priorización de sitios de conservación en la FVT, con áreas efectivas que detengan los procesos de CUS, podría conducir al mantenimiento de las ADG reales de las especies, y por lo tanto, de los procesos ecológicos y evolutivos (Escalante et al. 2020).

En este trabajo fue posible observar tendencias altitudinales en ADG históricas de las especies analizadas, mismas que se repitieron para las ADG reales (con CUS). En el caso de los efectos del cambio climático sobre las ADG, se ha documentado que las especies muestran cierta tendencia a trasladarse a mayores altitudes, así como a mayores latitudes (Parmesan y Yohe 2003; Colwell et al. 2008; Villers-Ruiz y Castañeda-Aguado 2013; Lara-Resendiz et al. 2019). Aunque es posible que el CUS no muestre un pa- trón específico, en general, las altitudes intermedias fueron las más afectadas. Algunos trabajos han encontrado que la altitud pudiera estar relacionada de manera directa con la deforestación en México, donde a mayor altitud, aumenta la pérdida de bosque (Mas et al. 2002; Pineda-Jaimes et al. 2009), aunque no es del todo claro si se trata de una combinación altitud-pendiente (Mas et al. 1996). Dentro de la FVT, por abajo de la cota altitudinal de los 2,800 m, en general se observa marcadamente el efecto del deterioro por actividades antrópicas (Villers et al. 2006).

La FVT presenta un relieve diverso (Ferrusquía-Villafranca 2007). En las zonas montañosas se ha demostrado que, dada la migración de las especies por el cambio climático, se requiere que se establezca conectividad entre relictos de vegetación para evitar pérdidas extremas en la biodiversidad (Challenger 2001). Sin embargo, algunas especies no encontrarán las condiciones ecológicas óptimas, por lo que se reducirá su tasa de supervivencia (Kaeslin et al. 2012; Villers-Ruiz y Castañeda-Aguado 2013). Existen diversos estudios del desplazamiento de las distribuciones geográficas de distintas especies debido al cambio climático (Pounds et al. 2005; Seimon et al. 2007; Raxworthy et al. 2008; Chen et al. 2009). Por el contrario, los estudios de los cambios en los patrones de distribución de las especies debidas al CUS son escasos. En México ya se ha previsto la selección de áreas de conservación que permita tomar decisiones tempranas sobre las modificaciones en las ADG debidas al cambio climático (Hannah et al. 2007; Jiménez-García y Peterson 2019); pero existen pocos trabajos sobre prioridades de conservación con base en escenarios prospectivos de CUS (por ejemplo: Contreras-Medina et al. 2010; Mendoza-Ponce et al. 2020).

En este trabajo partimos de la premisa de que cualquier CUS puede actuar negativamente sobre la distribución geográfica de las especies. En cambio, algunos autores consideran que los ambientes antropizados sub-óptimos pueden ser ocupados y utilizados por las especies (ver ejemplos en Ramírez-Bautista y Pineda-López 2016). Existe la posibilidad de que las ADG reales estén siendo sub-estimadas, al considerar que todas las áreas con CUS ya no están ocupadas por las especies aquí analizadas. No obstante, en términos de conservación, en México se ha documentado que los CUS modifican la efectividad de las áreas protegidas (Figueroa y Sánchez-Cordero 2008; Sahagún-Sánchez y Reyes-Hernández 2018. Los retos del futuro cercano deben enfocarse en el estudio de los múltiples efectos del CUS y desarrollar los mecanismos para paliarlos.

\section{CONCLUSIONES}

En general, las especies endémicas no estuvieron uniformemente distribuidas a lo largo de la FVT, mostrando diferentes comportamientos espaciales de magnitud, extensión, situación geográfica y continuidad. Además, existió diferenciación entre los grupos taxonómicos analizados. Los resultados sugieren que existen patrones distribucionales diversos dentro de la FVT, lo cual confirma su comple- 
jidad biótica, ecológica, evolutiva y geológica. Las ADG de las especies se concentraron en altitudes intermedias, en donde también ocurrió la mayor pérdida de área por el cambio de uso de suelo. Estas especies están enfrentando grave pérdida de su distribución histórica, lo cual podría estar obligándolas a habitar en condiciones sub-óptimas. Por lo anterior, los estudios areográficos sobre las ADG históricas y reales serán herramientas útiles para la preservación de áreas no transformadas que cumplan con el objetivo de prevenir la posible extirpación de las especies.

\section{AGRADECIMIENTOS}

Agradecemos los comentarios de dos revisores anónimos, los cuales fueron útiles para mejorar este trabajo. Esta investigación fue realizada gracias al Programa UNAM-DGAPA-PAPIIT, Proyecto IN217717. Michelle Farfán Gutiérrez y Gerardo Rodríguez-Tapia nos asesoraron en los análisis de CUS. Este trabajo se lo dedicamos al Dr. Juan José Morrone, digno sucesor del primer areógrafo de América, Eduardo Rapoport. El Dr. Morrone ha sido mentor y amigo de nuestro grupo de investigación desde hace más de 20 años ¡Gracias por tanto!

\section{LIERATURA CITADA}

Aguilar-Tomasini, A., T. Escalante, y M. Farfán. 2020. Effectiveness of natural protected areas for preventing land use and land cover changes of the Transmexican Volcanic Belt, Mexico. Regional Environmental Change, 20: 84.

Arriaga, L., C. Aguilar, D. Espinosa y R. Jiménez (Eds.). 1997. Regionalización ecológica y biogeográfica de México. Taller de la Comisión Nacional para el Conocimiento y Uso de la Biodiversidad. Conabio, Ciudad de México.

Arriaga, L., J.M. Espinoza, C.Aguilar, E. Martínez, L. Gómez y E. Loa. 2000. Regiones Prioritarias Terrestres de México. Comisión Nacional para el Conocimiento y Uso de la Biodiversidad, Ciudad de México.

Bonilla-Moheno, M. y T.M. Aide. 2020. Beyond deforestation: Land cover transitions in Mexico, Agricultural Systems, 178: 102734.

Cabrera, A.L. y A. Willink. 1973. Biogeografia de América Latina. Secretaría General de la Organización de los Estados Americanos, Serie de Biología, monografía 13, Washington, D. C.

Cantellano, E. 2007. Reconocimiento espacial de los paisajes. (pp. 39-55). En: Luna-Vega, I., J.J. Morrone y D. Espinosa (Eds.). Biodiversidad de la Faja Volcánica Transmexicana. Universidad Nacional Autónoma de México-Comisión Nacional para el Conocimiento y Uso de la Biodiversidad, Ciudad de México.

Challenger, A. 1998. Utilización y conservación de los ecosistemas terrestres de México: Pasado, presente y futuro. Conabio, UNAM y Sierra Madre, Ciudad de México.

Challenger, A. 2001. Estrategias para la conservación de ecosistemas. Gaceta ecológica, (61): 22-29.
Chen, I., H. Shiu, J. Holloway, V. Cheye, H. Barlow, J. Hill y C. Thomas. 2009. Elevation increases in moth assemblages over 42 years on a tropical mountain. Proceedings of the National Academy of Sciences of the United States of America, 106: 1479-1483.

Colwell, R.K., G. Brehm, C.L. Cardelus, A.C. Gilman y T.T. Longino. 2008. Global warming, elevational range shifts, and lowland biotic attrition in the wet tropics. Science, 322: 258-261.

Contreras-Medina R, Luna-Vega I, Ríos-Muñoz CA. 2010. Distribución de Taxus globosa (Taxaceae) en México: Modelos ecológicos de nicho, efectos del cambio de uso de suelo y conservación. Revista Chilena de Historia Natural, 83: 421-433.

Corral-Rosas, V. y J.J. Morrone. 2017. Analyzing the assembly of cenocrons in the Mexican transition zone through a time-sliced cladistic biogeographic analysis. Australian Systematic Botany, 29: 489-501. doi:10.1071/ sb16048.

Daskalova, G.N., I.H. Myers-Smith, A.D. Bjorkman, S.A. Blowes, S.R. Supp, A.E. Magurran y M. Dornelas. 2020. Landscape-scale forest loss as a catalyst of population and biodiversity change. Science, 368(6497): 1341-1347.

Escalante, T. 2015a. Modelos de distribución de especies de mamiferos y suculentas de la Faja Volcánica Transmexicana. Universidad Nacional Autónoma de México, Facultad de Ciencias. Informe final SNIB-Conabio, proyecto No. JM055. Ciudad de México.

Escalante, T. 2015b. Parsimony Analysis of Endemicity and Analysis of Endemicity: A fair comparison. Systematics and Biodiversity, 13(5): 413-418.

Escalante, T. 2017. A natural regionalization of the world based on primary biogeographic homology of terrestrial mammals. Biological Journal of the Linnean Society, 120: 349-362.

Escalante, T., A.M. Varela-Anaya, E.A. Noguera-Urbano, L.M. Elguea-Manrique, L.M. Ochoa-Ochoa, A.L. Gutiérrez-Velázquez, P. Reyes-Castillo, H.M. Hernández, C. Gómez-Hinostrosa, A.G. Navarro-Sigüenza, O. TéllezValdés y C. Rodríguez-Soto. 2020. Evaluation of five taxa as surrogates for conservation prioritization in the Transmexican Volcanic Belt, Mexico. Journal for Nature Conservation, 54: 125800.

Espinosa, D., S. Ocegueda, O. Flores-Villela, C. Aguilar-Zúñiga y J. Llorente-Bousquets. 2008. El conocimiento biogeográfico de las especies y su regionalización natural. (pp. 33-65). En: Sarukhán, J. (Ed.). Capital Natural de México Vol. 1. Comisión Nacional para el uso y Conocimiento de la Biodiversidad, Ciudad de México.

Fattorini, S. 2017. Endemism in historical biogeography and conservation biology: concepts and implications. Biogeographia, 32: 47-75.

Ferrari, L., T. Orozco-Esquivel, V. Manea y M. Manea. 2012. The dynamic history of the Trans-Mexican Volca- 
nic Belt and the Mexico subduction zone. Tectonophysics 522-523: 122-149.

Ferrusquía-Villafranca, I. 2007. Ensayo sobre la caracterización y significación biológica. (pp. 7-24). En: Luna, I., J.J. Morrone y D. Espinosa (Eds.). Biodiversidad de la Faja Volcánica Transmexicana. UNAM, Ciudad de México.

Figueroa, F., y V. Sánchez-Cordero. 2008. Effectiveness of natural protected areas to prevent land. Biodiversity Conservation, 17: 3223-3240.

Flores-Villela, O., L. Canseco-Márquez y L. Ochoa-Ochoa. 2010. Geographic distribution and conservation of the herpetofauna of the highlands of Central Mexico. (pp. 302-321). En: Wilson, L.D., J.H. Towsend y J.D. Johnson (Eds.). Conservation of MesoAmerican amphibians and reptiles. Eagle Mountain Publishing, Eagle Mountain.

Foley, J.A., R. DeFries, G.P. Asner, C. Barford, G. Bonan, S.R. Carpenter,F. Stuart Chapin, M.T. Coe1, G. C. Daily, H.K. Gibbs, J.H. Helkowski, T. Holloway, E.A. Howard, C.J. Kucharik, C. Monfreda, J.A. Patz, I.C. Prentice, N. Ramankutty y P.K. Snyder. 2005. Global Consequences of Land Use. Science, 309, 570-574.

Gámez, N., T. Escalante, G. Rodríguez, M. Linaje y J.J. Morrone. 2012. Caracterización biogeográfica de la Faja Volcánica Transmexicana y análisis de los patrones de distribución de su mastofauna. Revista Mexicana de Biodiversidad, 83: 258-272.

Gao, Y., A. Ghilardi, J.F. Mas, A. Quevedo, J. Paneque-Gálvez y M. Skutsch. 2018. Assessing forest cover change in Mexico from annual MODIS VCF data (2000-2010). International Journal of Remote Sensing, 39(22): 79017918.

Gaston, K.J. 1996. Species-range-size distributions: patterns, mechanism and implications. Trends in Ecology and Evolution, 11: 197-201.

GBIF. 2017. Databases. Referencias en el Material Supplementario de Escalante et al. (2020). Fecha de consulta: 2017.

Gómez-Tuena A., M.T. Orozco-Esquivel y L. Ferrari. 2005. Petrogénesis ígnea de la Faja Volcánica Transmexicana. Boletín de la Sociedad Geológica Mexicana, 57(3): 227-283.

González-Ávila A., M. Villegas-Ríos, J.A. Pérez-Trejo, R. Contreras-Medina e I. Luna-Vega. 2018. Riqueza y endemismo de las especies de Clavariadelphus (Clavariadelphaceae: Gomphales) en la Faja Volcánica Transmexicana. Revista Mexicana de Biodiversidad, 89(4): 1176-1189.

Guisan, A., y N.E. Zimmermann. 2000. Predictive habitat distribution models in ecology. Ecological Modelling, 135: 147-186.

Hannah, L., G. Midgley, S. Andelman, M. Araújo, G. Hughes, E. Martínez-Meyer, R. Pearson y P. Williams. 2007. Protected area needs in a changing climate. Frontiers in Ecology and the Environment, 5: 131-138.
Hansen A.J., R.S. DeFries y W. Turner. 2012. Land use change and biodiversity. En: Gutman G., A.C. Janetos, C.O. Justice, E.F. Moran, J.F.Mustard, R.R.Rindfuss, D. Skole, B.L.Turner II y M.A. Cochrane (Eds.). Land change science: Remote sensing and digital image processing, vol 6. Springer, Dordrecht.

Hernández, H. y C. Gómez-Hinostrosa. 2011. Mapping the Cacti of Mexico. dh books, Milborne Port.

Hernández, H. y C. Gómez-Hinostrosa. 2015. Mapping the Cacti of Mexico: Part II. Mammillaria. dh books, Milborne Port.

Hijmans, R. J., Phillips, S., Leathwick, J., \& Elith, J. (2017). dismo: Species Distribution Modeling, version 1.0. Retrieved from https://CRAN.Rproject.org/package=dismo/. Accessed 1 January 2017.

Illoldi, P. y T. Escalante. 2008. De los modelos de nicho ecológico a las áreas de distribución geográfica. Biogeografía, 3: 7 -12.

INEGI. 2017a. Conjunto de datos vectoriales de la carta de Uso del suelo y vegetación serie VI. Conjunto $\mathrm{Na}$ cional. https://www.inegi.org.mx/app/biblioteca/ficha. html?upc=889463598459. Fecha de consulta: 2016

INEGI. 2017b. Guía para la interpretación de cartografia uso del suelo y vegetación escala 1:250,000, Serie VI. INEGI, Aguascalientes.

INEGI, Lugo-Hupb, J., R. Vidal-Zepeda, A. Fernández-Equiarte, A. Gallegos-García y J. Zavala-H. 19901992. Hipsometría, escala 1:4000000. Extraído de Hipsometría y Batimetría, I.1.1 Atlas Nacional de México. Instituto de Geografía, UNAM, Ciudad de México.

IUCN. 2019. Guidelines for Using the IUCN Red List Categories and Criteria. Version 14. Prepared by the Standards and Petitions Committee. http://www.iucnredlist. org/documents/RedListGuidelines.pdf.

Jiménez-García D. y A.T. Peterson 2019. Climate change impact in endangered cloud forest tree species in Mexico. Revista Mexicana de Biodiversidad, 90: e902781.

Kaeslin, E., I. Redmond y N. Dudley (Eds.). 2012. La fauna silvestre en un clima cambiante. FAO, Roma.

Kohlman, B. y S. Sánchez-Colón. 1984. Estudio aerográfico del género Bursera Jacq. ex L. (Burseraceae) en México: una síntesis de métodos. (pp. 45-120). En: Ezcurra, E. y B. Kohlman (Eds.). Métodos cuantitativos en la biogeografía. Instituto de Ecología A. C., Ciudad de México.

Lara-Resendiz R.A., P. Galina-Tessaro, A.G. Pérez-Delgadillo, J.H. Valdez-Villavicencio y F.R. Méndez-de la Cruz. 2019. Efectos del cambio climático en una especie de lagartija termófila de amplia distribución (Dipsosaurus dorsalis): un enfoque ecofisiológico. Revista Mexicana de Biodiversidad, 90: e902888.

Mas, J.F., V. Sorani, y R. Alvarez. 1996. Elaboración de un modelo de simulación del proceso de deforestación. Investigaciones Geográficas, (Es5): 43-57.

Mas, J.F., A. Velázquez, R. Castro y A. Schmitt. 2002. Una 
evaluación de los efectos del aislamiento, la topografía, los suelos y el estatus de protección sobre las tasas de deforestación en México. RA'EGA, 6: 61-73.

Mendoza-Ponce, A.V., R.O. Corona-Núñez, F. Kraxner y F. Estrada. 2020. Spatial prioritization for biodiversity conservation in a megadiverse country. Anthropocene, 32: 100267.

Moreno-Sánchez, R., J.M. Torres Rojo, D. Carver, L. Niknami y E. Clay. 2017. Exploration of Land-cover Changes 2000-2010 at the National Level in Mexico Using GlobeLand30 Data Sets. Realidad, datos y espacio: Revista Internacional de Estadística y Geografia, 8: $30-45$.

Morrone, J.J. 2014. Biogeographical regionalisation of the Neotropical region. Zootaxa, 3782: 1-110.

Morrone, J.J. 2015. Biogeographical regionalisation of the world: a reappraisal. Australian Systematic Botany, 28(2-3): 81-90.

Morrone JJ. 2017. Neotropical biogeography: Regionalization and evolution. CRC Press, Boca Raton.

Morrone, J.J. 2020. The Mexican Transition Zone: A natural biogeographic laboratory to study biotic assembly. Cham, Springer.

Morrone, J.J. y T. Escalante. 2016. Introducción a la biogeografia. Facultad de Ciencias, UNAM, Ciudad de México.

Morrone, J.J., T. Escalante y G. Rodríguez-Tapia. 2017. Mexican biogeographic provinces: Map and shapefiles. Zootaxa, 4277(2): 277-279.

Morrone, J.J., D. Espinosa-Organista, C. Aguilar-Zúñiga y J. Llorente-Bousquets. 1999. Preliminary classification of the Mexican biogeographic provinces: A parsimony analysis of endemicity based on plant, insect, and bird taxa. Southwestern Naturalist, 44: 508-515.

Navarro-Sigüenza, A.G., A.T. Peterson y A. Gordillo-Martínez. 2003. Museums working together: the atlas of the birds of Mexico. (pp. 207-225). En: Collar, N., C. Fisher y C. Feare (Eds.). Why museums matter: avian archives in an age of extinction. Bulletin British Ornithologists' Club Supplement 123A, Londres.

Noguera-Urbano, E.A. 2017 El endemismo: diferenciación del término, métodos y aplicaciones. Acta Zoológica Mexicana (n. s.), 33(1): 89-107.

Noguera-Urbano, E.A. e I. Ferro. (2018). Environmental factors related to biogeographical transition zones of areas of endemism of Neotropical mammals. Australian Systematic Botany, 30(6), 485-494.

Parmesan, C. y G. Yohe. 2003. A globally coherent fingerprint of climate change impacts across nature systems. Nature, 421: 37-42.

Peterson, A.T., A.G. Navarro-Sigüenza y A. Gordillo-Martínez. 2016. The development of ornithology in Mexico and the importance of access to scientific information. Archives of Natural History, 43: 294-304.

Pineda-Jaimes, N.B., J. Bosque-Sendra, M. Gómez-Delgado y W. Plata-Rocha. 2009. Análisis de cambio del uso del suelo en el Estado de México mediante sistemas de información geográfica y técnicas de regresión multivariantes: Una aproximación a los procesos de deforestación. Investigaciones geográficas, (69): 33-52.

Pounds, J.A., M.P. Fogden y K.L. Masters. 2005. Responses of natural communities to climate change in a highland tropical forest. (pp. 70-74). En: Lovejoy T. E. y L. Hannah. Climate change and biodiversity. Yale University Press, New Haven.

QGIS. 2018. Geographic Information System. Open Source Geospatial Foundation Project. https://qgis.org. Fecha de consulta: 2019.

R Core Team. (2017). R: A language and environment for statistical computing. R Foundation for Statistical Computing, Vienna, Austria. ISBN 3-900051-07-0. Retrieved from https://www.R-project.org/.

Ramírez-Bautista, A. y R. Pineda-López (Eds.). 2016. Fauna Nativa en Ambientes Antropizados. CONACYT-UAQ, Querétaro.

Rapoport, E. 1975. Areografia: Estrategias geográficas de las especies. Fondo de Cultura Económica, Ciudad de México.

Rapoport, E.H. y J.A. Monjeau. 2001. Areografía. (pp. 2330). En: Llorente-Bousquets, J. y J.J. Morrone (Eds.). Introducción a la biogeografía en Latinoamerica; Teorías,conceptos, métodos y aplicaciones. Las prensas de Ciencias, Facultad de Ciencias, UNAM, Ciudad de México.

Raxworthy, C.J., R. Pearson, N. Rabibisoa, A. Rakotondrazafy, J. Ramanamanjato, A.P. Raselimanana, S. Wu, R.A. Nussbam y D.A. Stone. 2008. Extinction vulnerability of tropical montane endemism from warming and upslope displacement: a preliminary appraisal for the highest massif in Madagascar. Global Change Biology, 14: 1703-1720.

Reyes-Castillo, P. y M.A. Morón-Ríos. 2005. Passalidae y Lucanidae (Coleoptera: Scarabaeoidae) de México. Instituto de Ecología A.C. Bases de datos SNIB Conabio Proyectos No. AA014 y K005-Passalidae. Ciudad de México.

Sahagún-Sánchez, F.J. y H. Reyes-Hernández. (2018). Impactos por cambio de uso de suelo en las áreas naturales protegidas de la región central de la Sierra Madre Oriental, México. CienciaUAT, 12(2): 6-21.

Sánchez-Cordero, V., P. Illoldi-Rangel, M. Linaje, S. Sarkar y A.T. Peterson. 2005. Deforestation and extant distributions of Mexican endemic mammals. Biological Conservation, 126: 465-473.

Sánchez-Cordero, V., P. Illoldi-Rangel, T. Escalante, F. Figueroa, G. Rodríguez, M. Linaje, T. Fuller, y S. Sarkar. 2009. Deforestation and biodiversity conservation in Mexico. (pp. 279-297). En: Columbus, A.M. y L. Kuznetsov (Eds.). Endangered species: New research. Nova Science Publishers, Inc. Nueva York.

Sánchez-Cordero, V., M. Munguía y A.T. Peterson. 2004. GIS-based predictive biogeography in the context of 
conservation (pp. 311-323). En: M.V. Lomolino y L.R. Heaney (Eds.). Frontiers of Biogeography: New directions in the geography of nature. Sinauer Press, Sunderland.

Seimon, T.A., A. Seimon, P. Daszak, S.R.P. Halloy, L.M. Schloegel, C.A. Aguilar, P. Sowell, A.D.Haytt, B. Konecky y J.E. Simmons. 2007. Upward range extension of Andean anurans and chrytridiomycosis to extreme elevations in response to tropical deglaciation. Global Change Biology, 13: 288-299.

SNIB-Conabio. 2017. Databases. Red Mexicana de Información sobre Biodiversidad. http://www.biodiversidad. gob.mx. Fecha de consulta: 4 de abril de 2017.

Soberón, J., L. Osorio-Olvera y T. Peterson. 2017. Diferencias conceptuales entre modelación de nichos y modelación de áreas de distribución. Revista Mexicana de Biodiversidad, 88, 437-441.

Suárez-Mota, M.E. y O. Téllez-Valdés. 2014. Red de áreas prioritarias para la conservación de la biodiversidad del Eje Volcánico Transmexicano analizando su riqueza florística y variabilidad climática. Polibotánica (38): 67-93.

Suárez-Mota, M.E., O, Téllez-Valdés y R. Lira-Saade. 2013. Una regionalización de la Faja Volcánica Transmexicana con base en su riqueza florística. Botanical Sciences, 91: 93-105.

Recibido: 30 abril 2021

Aceptado: 15 junio 2021
Téllez-Valdés, O. 2017. Distribución potencial de las especies Pinaceae (Pinus) y Fagaceae (Quercus) de México. Universidad Nacional Autónoma de México. FES Iztacala. Bases de datos SNIB-Conabio_Quercus, proyecto No. JM010. Ciudad de México.

Toledo, V. M., J. Carabias y C. González-Pacheco. 1989. La producción rural en México: Alternativas ecológicas. Universo Veintiuno, Ciudad de México.

Velázquez, A., E. Durán, I. Ramírez, J.-F. Mas, G. Bocco, G. Ramírez y J.-L. Palacio. 2003. Land use-cover change processes in highly biodiverse areas: the case of Oaxaca, Mexico. Global Environmental Change, 13: 175-184.

Villers-Ruiz, L. y D. Castañeda-Aguado. 2013. Species and plant community reorganization in the Trans-Mexican Volcanic Belt under climate change conditions. Journal of Mountain Sciences, 10: 923-931.

Villers, L., F. Rojas y P. Tenorio. 2006. Guía botánica del Parque Nacional Malinche Tlaxcala-Puebla. Universidad Nacional Autónoma de México, Ciudad de México.

Yañez-Arenas, C., S. Mandujano, E. Martínez-Meyer, A. Pérez-Arteaga y A. González-Zamora. 2012. Modelación de la distribución potencial y el efecto del cambio de uso de suelo en la conservación de los ungulados silvestres del Bajo Balsas, México. Therya, 3(1): 67-79.

Zunino, M. y C. Palestrini. 1991. El concepto de especie y la biogeografía. Anales de Biología (Biología Animal), 6: $85-88$.

Cuadro 1. Media $(\overline{\mathrm{x}})$ y desviación estándar $\left(\mathrm{s}^{2}\right)$ de la superficie ocupada (Sup.) en hectáreas para las especies de cada clase taxonómica. *Por ser una única especie, la media corresponde a la única medida y no se calculó la $\mathrm{s}^{2}$.

\begin{tabular}{|l|l|r|r|r|r|}
\hline Sup. & \multicolumn{1}{|c|}{ Amphibia } & \multicolumn{1}{c|}{ Aves* } & \multicolumn{1}{c|}{ Insecta } & \multicolumn{1}{c|}{ Magnoliopsida } & \multicolumn{1}{c|}{ Mammalia } \\
\hline$\overline{\mathrm{x}}$ & $2,257,093.37$ & $1,707,604.05$ & $502,383.94$ & $4,339,328.71$ & $4,847,293.66$ \\
\hline $\mathrm{s}^{2}$ & $4,628,574.37$ & - & $1,691,169.37$ & $8,690,088.29$ & $6,905,976.27$ \\
\hline
\end{tabular}

Cuadro 2. Promedio las magnitudes máxima lineal, latitudinal y longitudinal en hectáreas para cada clase taxonómica. *Por ser una única especie, el promedio corresponde a la única medida.

\begin{tabular}{|l|c|c|c|c|c|}
\hline \multicolumn{1}{|c|}{ Magnitud } & Amphibia & Aves* & Insecta & Magnoliopsida & Mammalia \\
\hline Lineal & $270,665.58$ & $638,581.69$ & $171,616.31$ & $386,660.42$ & $530,966.32$ \\
\hline Latitudinal & $162,010.031$ & $345,403.41$ & $75,582.91$ & $261,518.23$ & $395,344.18$ \\
\hline Longitudinal & $250,905.353$ & $612,701.45$ & $159,442.04$ & $348,906.66$ & $554,696.17$ \\
\hline
\end{tabular}


Cuadro 3. Propuesta de clasificación de tipos de áreas de distribución para México, basada en Morrone et al. (2017).

\begin{tabular}{|l|l|l|l|}
\hline Extensión & Situación geográfica & Continuidad & \multicolumn{1}{c|}{ Ocupación } \\
\hline 1. Regional & 1. Transicional & 1. Continua & 1. Amplia \\
2. Continental & 2. Neártico- Transicional & 2. Discontinua & 2. Restringida \\
& $\begin{array}{l}\text { 3. Neártico-Transicional-Neotropical } \\
\text { 4.Transicional-Neotropical }\end{array}$ & & \\
& 5. Neotropical & & \\
& & & \\
\hline
\end{tabular}

Cuadro 4. Porcentaje de ocupación en los intervalos altitudinales de las áreas de distribución geográfica históricas para cada clase taxonómica.

\begin{tabular}{|c|c|c|c|c|c|}
\hline $\begin{array}{l}\text { Intervalo altitudinal } \\
\text { (msnm) }\end{array}$ & Amphibia & Aves* & Insecta & Magnoliopsida & Mammalia \\
\hline 0 a 200 & 3.70 & 0.00 & 0.01 & 0.72 & 1.13 \\
\hline 200 a 500 & 4.48 & 0.00 & 0.16 & 1.30 & 1.17 \\
\hline 500 a 1000 & 5.14 & 0.01 & 0.74 & 2.91 & 2.48 \\
\hline 1,000 a 1,500 & 10.95 & 0.39 & 3.61 & 10.15 & 10.32 \\
\hline 1,500 a 2,000 & 31.44 & 5.15 & 11.43 & 27.00 & 28.96 \\
\hline 2,000 a 2,500 & 28.85 & 15.69 & 39.33 & 36.07 & 33.42 \\
\hline 2,500 a 3,000 & 12.77 & 52.96 & 36.74 & 18.40 & 17.66 \\
\hline 3,000 a 3,500 & 2.12 & 20.66 & 6.30 & 2.76 & 4.16 \\
\hline 3,500 a 4,000 & 0.46 & 4.53 & 1.39 & 0.58 & 0.59 \\
\hline 4,000 a 4,500 & 0.08 & 0.55 & 0.24 & 0.09 & 0.09 \\
\hline 4,500 a 5,000 & 0.01 & 0.06 & 0.03 & 0.01 & 0.01 \\
\hline$>5,000$ & 0.00 & 0.00 & 0.00 & 0.00 & 0.00 \\
\hline
\end{tabular}




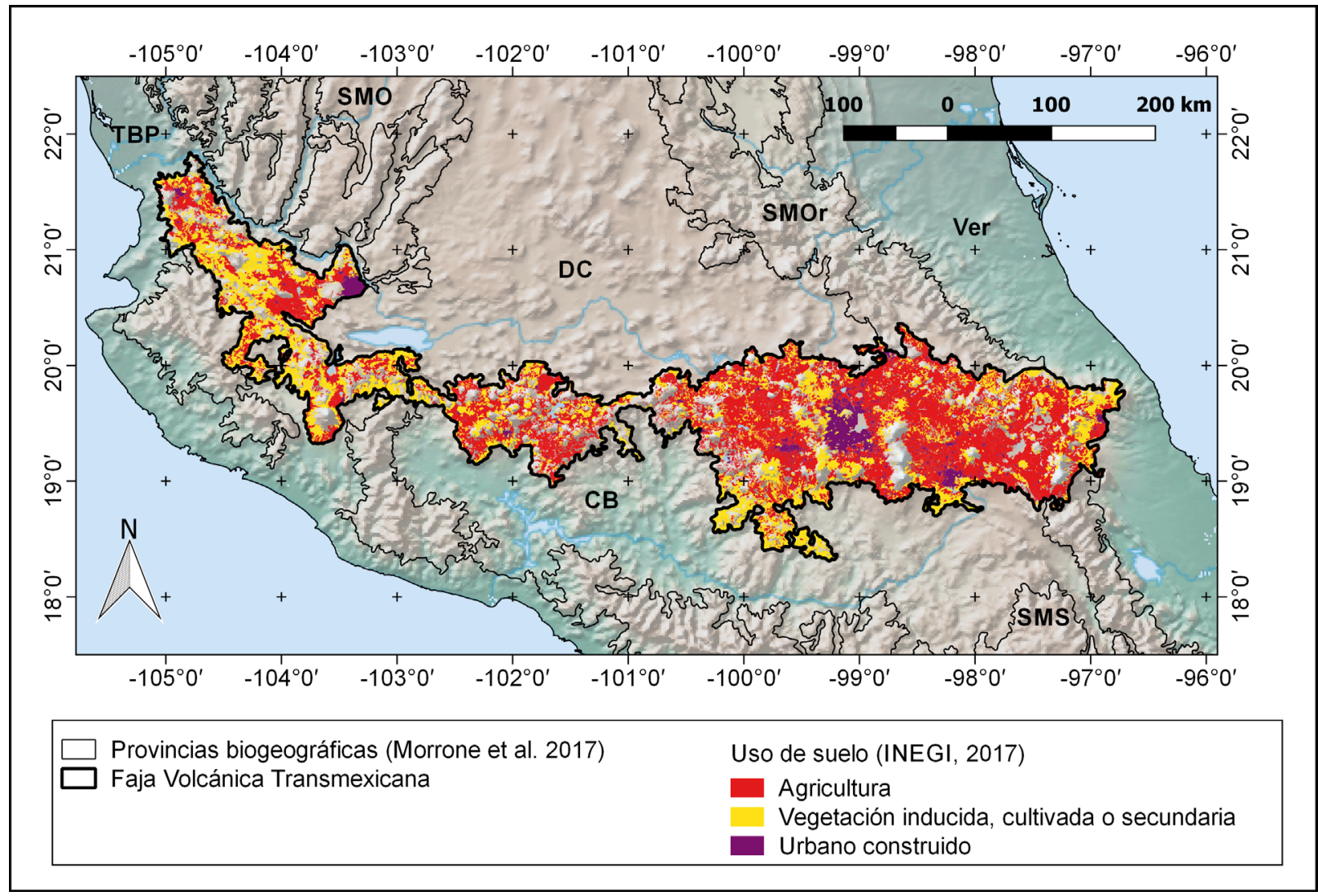

Fig. 1. Mapa de la localización de la Faja Volcánica Transmexicana con el cambio de uso de suelo de la Serie VI de Uso del Suelo y Vegetación (INEGI 2017a, 2017b). Provincias biogeográficas de Morrone et al. (2017): CB: Cuenca del Balsas, DC: Desierto Chihuahuense, SMO: Sierra Madre Occidental, SMOr: Sierra Madre Oriental, SMS: Sierra Madre del Sur, Ver: Veracruzana, TBP: Tierras Bajas del Pacífico.

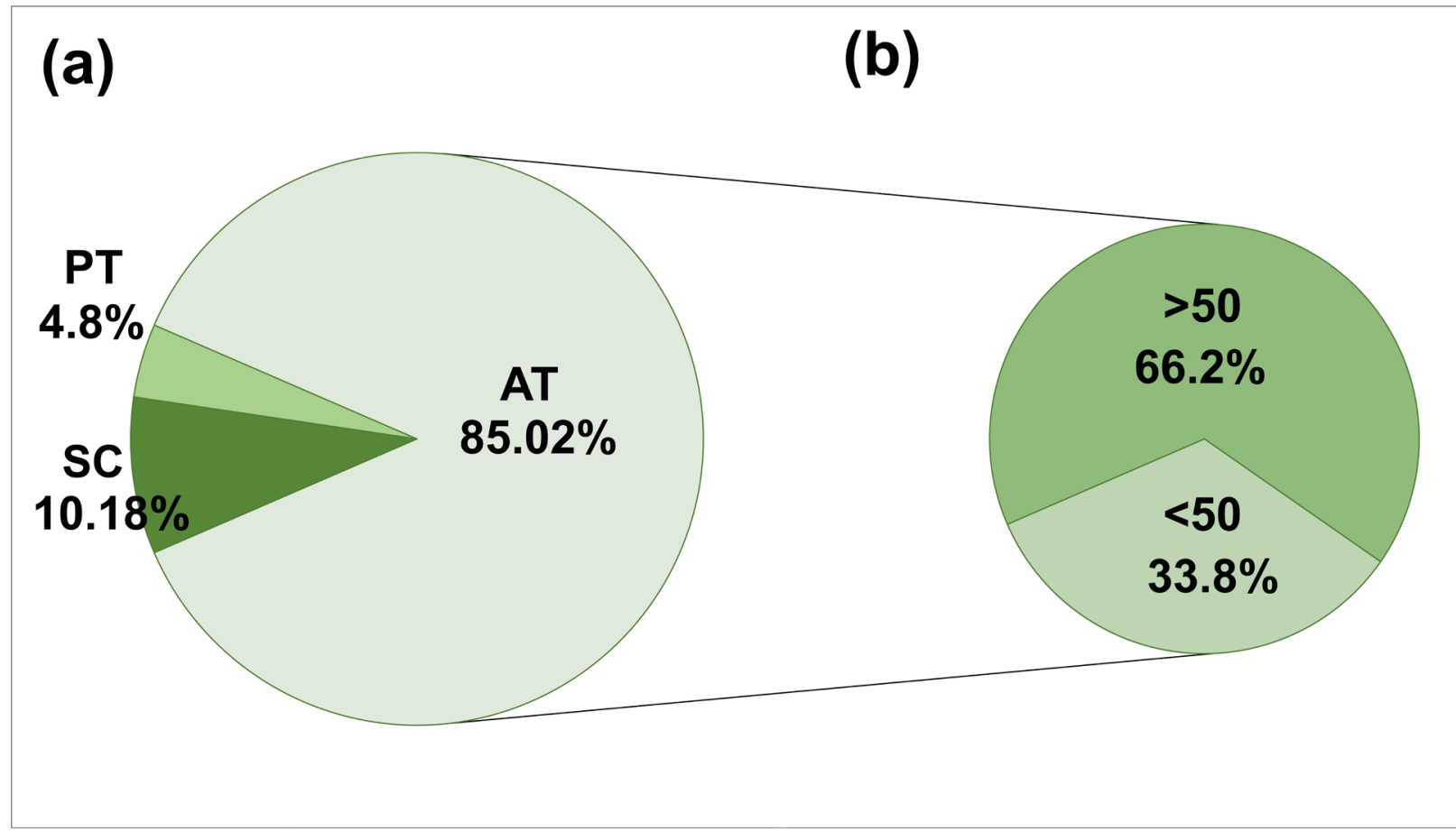

Fig. 2. Cambios en el porcentaje de las áreas de distribución histórica de 167 especies endémicas de la Faja Volcánica Transmexicana debidas al cambio de uso de suelo. (a) Todas las especies. PT: Pérdida total, SC: Sin cambio, AT: Área transformada. (b) Especies con área transformada con porcentaje de pérdida de área de $>50 \% \mathrm{y}<50 \%$. 


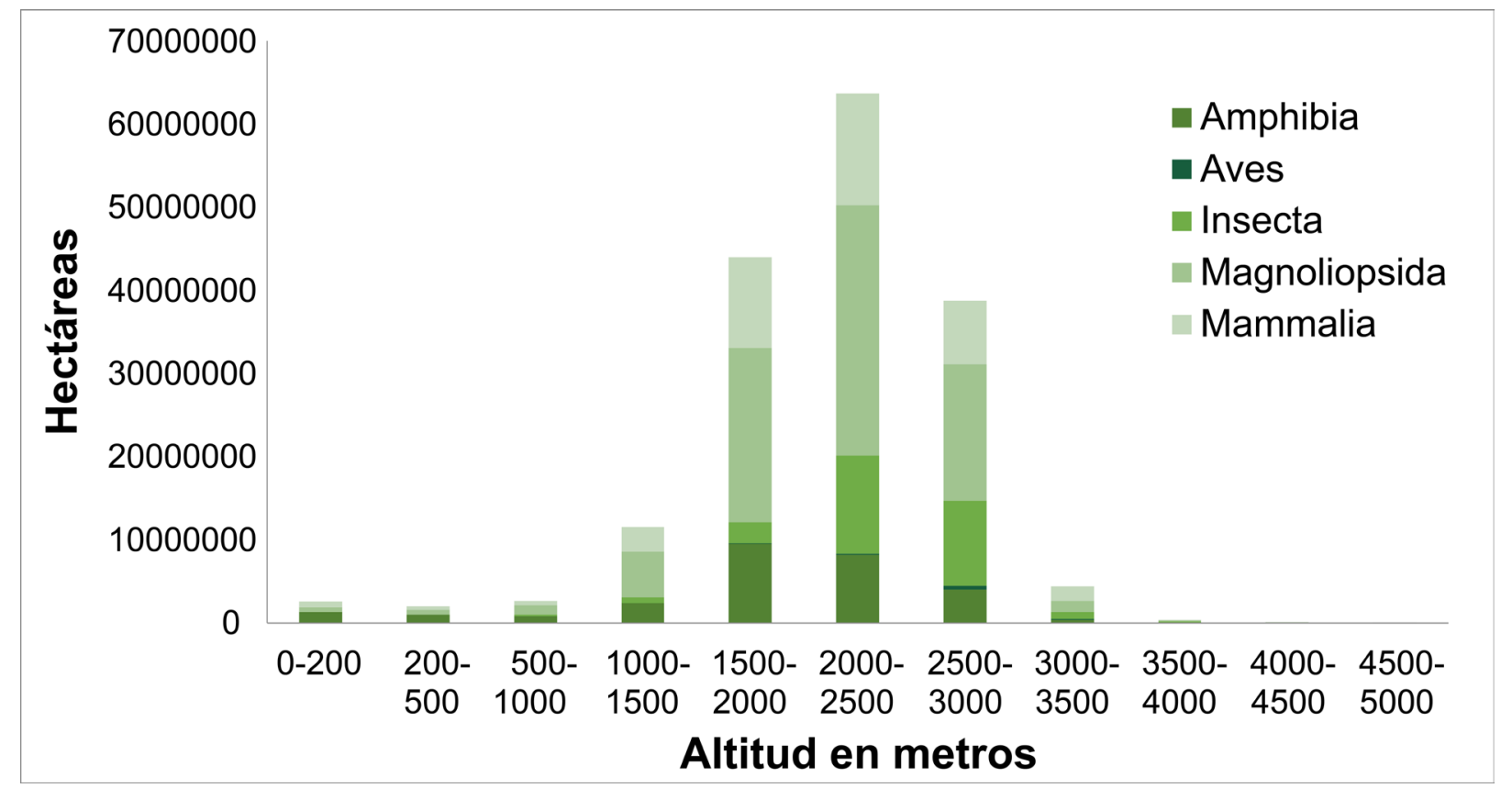

Fig. 3. Área perdida en hectáreas por intervalos altitudinales para cada clase taxonómica, con base en la hipsometría de INEGI (1990-1992) y en la Serie VI de Uso del Suelo y Vegetación (INEGI 2017a, 2017b).

Material suplementario

Lista de especies analizadas, con detalles sobre cuantificación de su área histórica y real, así como su clasificación de acuerdo con su extensión, situación geográfica, continuidad y ocupación. 